\title{
Lattice Boltzmann algorithms without cubic defects in Galilean invariance on standard lattices
}

\author{
Paul J. Dellar
}

OCIAM, Mathematical Institute, Radcliffe Observatory Quarter, Oxford, OX2 6GG, UK

\begin{abstract}
The vast majority of lattice Boltzmann algorithms produce a non-Galilean invariant viscous stress. This defect arises from the absence of a term in the third moment, the equilibrium heat flow tensor, proportional to the cube of the fluid velocity. This moment cannot be specified independently of the lower moments on the standard lattices such as D2Q9, D3Q15, D3Q19 or D3Q27. A partial correction has recently been demonstrated that restores some of these missing cubic terms on the D2Q9 and D3Q27 tensor product lattices. This correction restores Galilean invariance for shear flows aligned with the coordinate axes, but flows inclined at arbitrary angles may show larger errors than before. These remaining errors are due to the diagonal terms of the equilibrium heat flow tensor, which cannot be corrected on standard lattices. However, the remaining errors may be largely absorbed by introducing a matrix collision operator with velocity-dependent collision rates for the diagonal components of the momentum flux tensor. This completely restores Galilean invariance for flows with uniform density, and in general reduces the magnitude of the defect in Galilean invariance from Mach number cubed to Mach number to the fifth power. The effectiveness of the resulting algorithm is demonstrated by comparisons with the standard and partially corrected lattice Boltzmann algorithms for two- and three-dimensional flows.
\end{abstract}

Key words:

Galilean invariance, matrix collision operators, shear flows, computational fluid dynamics

Submitted 27 January 2013, accepted 17 November 2013.

Published as J. Comput. Phys. 259 270-283

\section{Introduction}

The Navier-Stokes equations may be derived from the Boltzmann equation that describes dilute monatomic gases. This derivation seeks slowly varying solutions using the Chapman-Enskog expansion $[1,2]$. This expansion involves only the lowest few moments of the Maxwelll-Boltzmann distribution. The lattice Boltzmann approach to computational fluid dynamics seeks to construct discrete kinetic theories that also yield the Navier-Stokes equations under the same limit. The derivations may proceed exactly in parallel if one replaces the integral moments of continuous kinetic theory with discrete moments or sums over a discrete velocity space.

The continuous Boltzmann equation expresses the evolution of the distribution function $f(\mathbf{x}, \boldsymbol{\xi}, t)$ for a dilute monatomic gas as

$$
\partial_{t} f+\boldsymbol{\xi} \cdot \nabla f=C[f, f] .
$$

The distribution function gives the number density of particles at position $\mathbf{x}$ and time $t$ moving with velocity $\boldsymbol{\xi}$. The left-hand side is a Lagrangian derivative along particle trajectories, while the integral collision operator on the right hand side involves only relative velocities between pairs of particles. The Boltzmann equation is thus invariant under Galilean transformations. This is commonly expressed in kinetic theory by working with the peculiar velocity $\mathbf{c}=\boldsymbol{\xi}-\mathbf{u}$, the difference between $\boldsymbol{\xi}$ and the local fluid velocity $\mathbf{u}$ defined in (6) below.

The lattice Boltzmann approach achieves computational efficiency by restricting the particle velocity $\boldsymbol{\xi}$ to a finite set $\left\{\boldsymbol{\xi}_{i}: i=\right.$ $0, \ldots, N-1\}$, and evolves the finite set of functions $f_{i}(\mathbf{x}, t)$ for $i=0, \ldots, N-1$ rather than $f(\mathbf{x}, \boldsymbol{\xi}, t)$. The corresponding discrete Boltzmann equation is a set of $N$ linear, constant coefficient, hyperbolic PDEs, such as (4) below, that is readily discretised in space and time. All nonlinearity is confined to an algebraic collision term, the discrete analogue of $C[f, f]$. However, the adoption of a finite velocity set $\left\{\boldsymbol{\xi}_{i}\right\}$ inevitably breaks Galilean invariance at the level of the discrete Boltzmann equation. The set of particle velocities typically satisfies the symmetry property that for each $i$ there is an $\bar{i}$ such that $\boldsymbol{\xi}_{i}+\boldsymbol{\xi}_{\bar{i}}=0$, and includes a so-called rest particle with velocity $\boldsymbol{\xi}_{0}=0$. The frame in which these two properties hold defines a preferred frame.

A much more serious concern is loss of Galilean invariance in the slowly varying limit. The common lattice Boltzmann formulations contain too few degrees of freedom to correctly reproduce the viscous stress in the Navier-Stokes equations. The common formulations use discrete equilibrium distributions that are quadratic polynomials in the fluid velocity $\mathbf{u}$ [3-5]

$$
f_{i}^{(0)}=w_{i} \rho\left(1+3 \mathbf{u} \cdot \boldsymbol{\xi}_{i}+\frac{9}{2}\left(\mathbf{u} \cdot \boldsymbol{\xi}_{i}\right)^{2}-\frac{3}{2}|\mathbf{u}|^{2}\right),
$$

where $\rho$ is the fluid density, and the $w_{i}$ are weights attached to the different particle velocities $\boldsymbol{\xi}_{i}$. The first three moments of these discrete equilibria coincide with the corresponding moments of the Maxwell-Boltzmann distribution, but the next 


$\begin{array}{rllll}\text { Speed } & \text { Velocity template } & \text { D3Q15 } & \text { D3Q19 } & \text { D3Q27 } \\ 0 & (0,0,0) & 2 / 9 & 1 / 3 & 8 / 27 \\ 1 & ( \pm 1,0,0) & 1 / 9 & 1 / 18 & 2 / 27 \\ \sqrt{2} & ( \pm 1, \pm 1,0) & 0 & 1 / 36 & 1 / 54 \\ \sqrt{3} & ( \pm 1, \pm 1, \pm 1) & 1 / 72 & 0 & 1 / 216\end{array}$

Table 1: The weights $w_{i}$ for the four different velocity shells $\left|\boldsymbol{\xi}_{i}\right|^{2} \in\{0,1,2,3\}$ for the three common 3D lattices [5, 7]. The particle velocities in each shell comprise the given form and its permutations. The D3Q15 lattice omits the velocities $( \pm 1, \pm 1,0)$ and their permutations, while the D3Q19 lattice omits the velocities $( \pm 1, \pm 1, \pm 1)$.

moment is lacking a $\rho \mathbf{u u u}$ term due to the truncation of the discrete equilibria at $O\left(|\mathbf{u}|^{2}\right)$. This leads to the viscous momentum flux [6]

$$
\mathbf{\Pi}^{(1)}=-\tau \rho \theta\left[(\nabla \mathbf{u})+(\nabla \mathbf{u})^{\top}\right]+\tau \nabla \cdot(\rho \mathbf{u u u})
$$

The first term is a Newtonian viscous stress with dynamic viscosity $\mu=\tau \rho \theta$, where $\theta$ is the temperature, but the second term $\tau \nabla \cdot(\rho \mathbf{u u u})$ is an error term that breaks Galilean invariance. The ratio of the two terms scales as $|\mathbf{u}|^{2} / \theta=\mathrm{Ma}^{2}$, so the error term is commonly treated as negligible on the assumption that the Mach number Ma is sufficiently small. However, it is an obstacle to the accuracy of the lattice Boltzmann approach at finite Ma, and we show in Sec. 8 below that it has a detrimental effect on numerical stability.

The natural remedy to this loss of Galilean invariance is to restore the missing $O\left(|\mathbf{u}|^{3}\right)$ terms to the discrete equilibria, but this is not possible when the particle velocities form one of the standard lattices known as D2Q9, D3Q15, D3Q19 or D3Q27 $[5,7]$. The particle velocities $\boldsymbol{\xi}_{i}$ for the three different 3D lattices are given in Table 1, while the velocities for the D2Q9 lattice arise from projecting any of the 3D lattices onto the $x y$ plane. The dimensionless particle velocity components $\xi_{i \alpha}$ all lie in the set $\{-1,0,1\}$, so $\xi_{i \alpha}^{3}=\xi_{i \alpha}$ and $\sum_{i} \xi_{i \alpha}^{3} f_{i}=\sum_{i} \xi_{i \alpha} f_{i}$. The third moment that appears in the calculation of the viscous stress thus cannot be adjusted independently of the first moment.

The next simplest approach is to adopt a larger lattice with more discrete particle velocities [8-10], although a relatively early attempt [11] to restore the cubic terms on a two-dimensional 17 velocity lattice used incorrect equilibria [12]. Besides adding additional particle velocities whose components lie in a larger integer set such as $\{-2,-1,0,1,2\}$, one may use higher order Gauss-Hermite quadratures to motivate other particle velocities whose components are not integer multiples of each other [13-15]. The latter require interpolation of distribution functions between lattice points or some alternative space-time discretisation, while any addition of more degrees of freedom per lattice point increases computational cost, and introduces more scope for grid-scale instabilities of the type analysed in [16].

The free energy approach of Swift et al. [17] simulates non-ideal fluids by adding terms proportional to the density gradient to the equilibrium momentum flux. These gradients are calculated from finite difference approximations on the lattice. Further finite difference approximations to velocity gradients may also be added to cancel the non-Galilean-invariant defects introduced by the non-ideal terms [18, 19], and also those due to the underlying ideal fluid [20]. However, this again increases complexity and scope for grid-scale instabilities. The standard lattice Boltzmann space/time discretisation (see Sec. 5) defines effective finite difference stencils that differ from those commonly used [21]. Any inconsistency between the two sets of finite difference stencils may create grid-scale artifacts.

More recently, it has been realised that a partial correction of the third moment is possible on the D2Q9 lattice [12, 22-24]. This restores Galilean invariance for shear flows aligned with the coordinate axes, which depend only upon the $\Pi_{x y}$ component of the momentum flux, but errors remain in the diagonal components $\Pi_{x x}$ and $\Pi_{y y}$. In this paper we show that these remaining errors may be largely absorbed using a nonlinear matrix collision operator with relaxation times that depend upon the local fluid velocity $\mathbf{u}$. Matrix collision operators originally appeared as linearisations of lattice gas binary collision operators [25], but have since been developed to improve the stability of lattice Boltzmann algorithms while preserving the isotropy of the viscous stress [26-29]. In fact, the D3Q13 algorithm requires a matrix collision operator to produce an isotropic viscous stress [30]. However, they have also been used to model the strongly anisotropic stress-strain relation that holds in strongly magnetised plasmas [31]. In this paper we introduce small intentional anisotropies into the matrix collision operator to correct the anisotropies caused by the partial restoration of the cubic terms in the tensor $\mathbf{Q}^{(0)}$. The resulting algorithm completely restores Galilean invariance at the Navier-Stokes level for flows with uniform density, and reduces the error in the viscous stress from $O\left(\mathrm{Ma}^{3}\right)$ to $O\left(\mathrm{Ma}^{5}\right)$ in the presence of density variations.

\section{The viscous stress in lattice Boltzmann hydrodynamics}

Lattice Boltzmann algorithms are space-time discretisations of discrete Boltzmann equations of the form

$$
\partial_{t} f_{i}+\boldsymbol{\xi}_{i} \cdot \nabla f_{i}=-\sum_{j=0}^{N-1} \Omega_{i j}\left(f_{j}-f_{j}^{(0)}\right) .
$$

Each $f_{i}(\mathbf{x}, t)$ is a particle distribution function that propagates with velocity $\boldsymbol{\xi}_{i}$, and the $\Omega_{i j}$ are the components of an $N \times N$ collision matrix $\boldsymbol{\Omega}$. The equilibrium distributions $f_{j}^{(0)}$ are prescribed functions of the moments $\rho$ and $\mathbf{u}$ defined in (6) below. For example, the common quadratic polynomial equilibria are given in (2). 
The discrete Boltzmann equation (4) implies the evolution equations

$$
\partial_{t} \rho+\nabla \cdot(\rho \mathbf{u})=0, \quad \partial_{t}(\rho \mathbf{u})+\nabla \cdot \boldsymbol{\Pi}=0, \quad \partial_{t} \boldsymbol{\Pi}+\nabla \cdot \mathbf{Q}=-\frac{1}{\tau}\left(\boldsymbol{\Pi}-\mathbf{\Pi}^{(0)}\right)
$$

for moments of the $f_{i}$,

$$
\rho=\sum_{i=0}^{N-1} f_{i}, \quad \rho \mathbf{u}=\sum_{i=0}^{N-1} \boldsymbol{\xi}_{i} f_{i}, \quad \boldsymbol{\Pi}=\sum_{i=0}^{N-1} \boldsymbol{\xi}_{i} \boldsymbol{\xi}_{i} f_{i}, \quad \mathbf{Q}=\sum_{i=0}^{N-1} \boldsymbol{\xi}_{i} \boldsymbol{\xi}_{i} \boldsymbol{\xi}_{i} f_{i} .
$$

The first few moments determine the fluid density $\rho$, velocity $\mathbf{u}$, and momentum flux $\boldsymbol{\Pi}$. The superscript $(0)$ on $\boldsymbol{\Pi}^{(0)}$ indicates a moment of the equilibrium distributions $f_{i}^{(0)}$. The right hand side (5) relies on $\xi_{i} \xi_{i}$ being a left eigenvector of the collision matrix $\boldsymbol{\Omega}$ with eigenvalue $1 / \tau$. The simplest choice is the Bhatnagar-Gross-Krook [32] collision matrix $\boldsymbol{\Omega}=(1 / \tau) \mathbf{l}$, but more complicated choices with multiple eigenvalues (multiple relaxation times) may be used to improve stability [26-29].

The same system (5) arises as the first few members of an infinite hierarchy of moment equations for the continuous Boltzmann equation (1). Boltzmann's binary collision operator $C[f, f]$ drives $f$ towards the Maxwell-Boltzmann equilibrium distribution

$$
f^{(0)}=\rho(2 \pi \theta)^{-3 / 2} \exp \left(-|\mathbf{u}-\boldsymbol{\xi}|^{2} /(2 \theta)\right)
$$

while conserving mass, momentum, and energy. The quadratic polynomial equilibria (2) may be constructed by seeking a truncated expansion in tensor Hermite polynomials whose first three moments coincide with those of the continuous $f^{(0)}$ above when the temperature $\theta$ takes the value $1 / 3$ in the dimensionless lattice units in which the particle velocity components lie in the set $\{-1,0,1\}$.

The Navier-Stokes equations arise from seeking solutions to the moment hierarchy (5) that vary on a slow timescale $T$ much longer than the collisional timescale $\tau$. The modern Chapman-Enskog expansion [1,2] uses a multiple-scales expansion of the non-conserved moments and the time derivative,

$$
\partial_{t}=\partial_{t_{0}}+\epsilon \partial_{t_{1}}+\cdots, \quad \boldsymbol{\Pi}=\boldsymbol{\Pi}^{(0)}+\epsilon \boldsymbol{\Pi}^{(1)}+\epsilon^{2} \boldsymbol{\Pi}^{(2)}+\cdots, \quad \mathbf{Q}=\mathbf{Q}^{(0)}+\epsilon \mathbf{Q}^{(1)}+\epsilon^{2} \mathbf{Q}^{(2)}+\cdots,
$$

in the small parameter $\epsilon=\tau / T$, which is equivalent to the Knudsen number. The conserved moments $\rho$ and $\mathbf{u}$ are left unexpanded, equivalent to imposing solvability conditions on an expansion of $f=f^{(0)}+\epsilon f^{(1)}+\cdots$. The combination of the expansion of $\partial_{t}$ and the solvability conditions prevents the expansion of $f$ from becoming disordered at long times when $t \sim T / \epsilon$, as arises under the Hilbert expansion of $f$ alone [33].

At leading order we obtain the compressible Euler equations

$$
\partial_{t_{0}} \rho+\nabla \cdot(\rho \mathbf{u})=0, \quad \partial_{t_{0}}(\rho \mathbf{u})+\nabla \cdot \boldsymbol{\Pi}^{(0)}=0,
$$

where the inviscid momentum flux $\mathbf{\Pi}^{(0)}=\theta \rho \mathbf{I}+\rho \mathbf{u u}$, with $\mathbf{I}$ the identity tensor. Evaluating the last of (5) at leading order gives

$$
\partial_{t_{0}} \mathbf{\Pi}^{(0)}+\nabla \cdot \mathbf{Q}^{(0)}=-\frac{1}{T} \mathbf{\Pi}^{(1)}
$$

where $\mathbf{Q}^{(0)}$ is known from $f^{(0)}$. Evaluating $\partial_{t_{0}} \mathbf{\Pi}^{(0)}=\partial_{t_{0}}(\theta \rho \mathbf{l}+\rho \mathbf{u u})$ using the Euler equations (9) gives

$$
\partial_{t_{0}} \Pi_{\beta \gamma}^{(0)}=-\theta \delta_{\beta \gamma} \nabla \cdot(\rho \mathbf{u})-\theta u_{\beta} \frac{\partial \rho}{\partial x_{\gamma}}-\theta \frac{\partial \rho}{\partial x_{\beta}} u_{\gamma}-\partial_{\alpha}\left(\rho u_{\alpha} u_{\beta} u_{\gamma}\right),
$$

using Greek indices for Cartesian tensor components. The third integral moment of the Maxwell-Boltzmann distribution (7) has components

$$
Q_{\alpha \beta \gamma}^{(0)}=\int \xi_{\alpha} \xi_{\beta} \xi_{\gamma} f^{(0)} d \boldsymbol{\xi}=\rho u_{\alpha} u_{\beta} u_{\gamma}+\rho \theta\left(u_{\alpha} \delta_{\beta \gamma}+u_{\beta} \delta_{\gamma \alpha}+u_{\gamma} \delta_{\alpha \beta}\right)
$$

Substituting this expression into (10) gives the Newtonian viscous stress for an isothermal fluid,

$$
\mathbf{\Pi}^{(1)}=-\tau \rho \theta\left[(\nabla \mathbf{u})+(\nabla \mathbf{u})^{\top}\right] .
$$

However, the quadratic polynomial equilibria (2) used by the most common lattice Boltzmann algorithms have a third moment $\mathbf{Q}^{(0)}$ that omits the first term $\rho u_{\alpha} u_{\beta} u_{\gamma}$ compared with (12). This missing term is responsible for the error term $\tau \nabla \cdot(\rho \mathbf{u u u})$ in the viscous momentum flux (3) that leads to loss of Galilean invariance [6]. In the following we show that this error term may be greatly reduced by using a suitable matrix collision operator $\mathbf{\Omega}(\mathbf{u})$ that depends upon the local fluid velocity.

\section{Partially corrected third moment}

The natural remedy is to restore the missing cubic terms to $f_{i}^{(0)}$ so that the discrete third moment $\mathbf{Q}^{(0)}$ coincides with (12) for the Maxwell-Boltzmann distribution. However, the commonly used lattices such as D2Q9, D3Q15, D3Q19, and D3Q27 do not have enough degrees of freedom to specify the whole $\mathbf{Q}^{(0)}$ tensor independently of the lower moments. The particle velocity 
components $\xi_{i \alpha} \in\{-1,0,1\}$ for these lattices so, for example, $\xi_{i x}^{3}=\xi_{i x}$, which implies $Q_{x x x}^{(0)}=\rho u_{x}$. It is possible to correct the off-diagonal components of $\mathbf{Q}^{(0)}$ on the D2Q9 lattice [12, 22, 23]

$$
Q_{x x y}^{(0)}=\theta \rho u_{y}+\rho u_{x}^{2} u_{y}, \quad Q_{x y y}^{(0)}=\theta \rho u_{x}+\rho u_{x} u_{y}^{2}
$$

in agreement with (12), while $Q_{x x x}^{(0)}$ and $Q_{y y y}^{(0)}$ must remain unchanged. Wagner \& Yeomans [22] constructed equilibria that are consistent with the Galilean transformations parallel to the coordinate axes that they used to implement Lees-Edwards sliding periodic boundary conditions. These equilibria satisfy the conditions (14). The same partial corrections were later rediscovered by evaluating cubic equilibria previously proposed for the D1Q5 and D2Q17 lattices on the D2Q9 lattice [11, 12, 23]. The conditions (14) are also satisfied by the truncated expansion for $|\mathbf{u}| \ll 1$ of certain entropy-minimising equilibria [24]. The $x y$ component of the viscous stress is now correct, which is sufficient for simulating axis-aligned shear flows. For example, the evolution of the velocity field $\mathbf{u}=u(x, t) \hat{\mathbf{y}}$ is given by

$$
\partial_{t}(\rho u)+\partial_{x} \Pi_{x y}=0
$$

in which only $\Pi_{x y}$ plays a rôle. However, non-Galilean invariant terms still remain in the $\Pi_{x x}$ and $\Pi_{y y}$ components, which become visible in more general flows, such as the linear shear flows inclined to the axes studied in Sec. 6

To see why a partial, but only a partial, correction of $\mathbf{Q}^{(0)}$ is possible on the D2Q9 lattice, it is useful to consider a basis of moments for this lattice. The first three tensor Hermite polynomials, $1, \boldsymbol{\xi}_{i}, \boldsymbol{\xi}_{i} \boldsymbol{\xi}_{i}-\theta \mathbf{l}$ may be completed with the quantities $\boldsymbol{\xi}_{i} g_{i}$ and $g_{i}=(1,-2,-2,-2,-2,4,4,4,4)$ to form a basis that is orthogonal with respect to the weighted inner product [16, 29]

$$
\left\langle f_{i}, h_{i}\right\rangle=\sum_{i=0}^{8} w_{i} f_{i} h_{i}
$$

with the same weights $w_{i}$ that appear in the equilibria (2) The additional basis elements may be written as $g_{i}=\left|\xi_{i}\right|^{4}-(15 / 9)\left|\xi_{i}\right|^{2}+$ $2 / 9$ and $\boldsymbol{\xi}_{i} g_{i}=\boldsymbol{\xi}_{i}\left(\left|\boldsymbol{\xi}_{i}\right|^{2}-4 / 3\right)$, as constructed by orthogonalising $\left|\boldsymbol{\xi}_{i}\right|^{2}$ and $\boldsymbol{\xi}_{i}\left|\boldsymbol{\xi}_{i}\right|^{2}$ against the three tensor Hermite polynomials using the Gram-Schmidt algorithm. This construction shows that there are only two independent degrees of freedom in the moments involving $\boldsymbol{\xi}_{i}^{3}$, since the most general equilibria for the D2Q9 lattice that still reproduce the Euler equations may be written as $[16,29]$

$$
f_{i}^{(0)}=w_{i}\left[\rho+3 \rho \mathbf{u} \cdot \boldsymbol{\xi}_{i}+\rho \mathbf{u u}:\left(\boldsymbol{\xi}_{i} \boldsymbol{\xi}_{i}-\frac{1}{3} \mathbf{I}\right)+\frac{1}{4} g_{i} N^{(0)}+\frac{3}{8} g_{i} \xi_{i} \cdot \mathbf{J}^{(0)}\right]
$$

for arbitrary values of the scalar $N^{(0)}$ and vector $\mathbf{J}^{(0)}$. In particular, the equilibria with the partial cubic corrections (14) may be written as

$$
f_{i}^{(0)}=w_{i} \rho\left[1+3 \mathbf{u} \cdot \boldsymbol{\xi}_{i}+\frac{9}{2}\left(\mathbf{u} \cdot \boldsymbol{\xi}_{i}\right)^{2}-\frac{3}{2}|\mathbf{u}|^{2}+\frac{27}{2}\left(\left|\boldsymbol{\xi}_{i}\right|^{2}-\frac{4}{3}\right)\left(u_{x}^{3} \xi_{i x}+u_{y}^{3} \xi_{i y}\right)\right] \text {. }
$$

\section{Further correction using a matrix collision operator}

The viscous stress for a general discrete equilibrium third moment $\mathbf{Q}^{(0)}$ may be written as

$$
\mathbf{\Pi}^{(1)}=-\tau \rho \theta\left[(\nabla \mathbf{u})+(\nabla \mathbf{u})^{\top}\right]-\tau \nabla \cdot\left(\Delta \mathbf{Q}^{(0)}\right)
$$

where $\Delta \mathbf{Q}^{(0)}=\mathbf{Q}^{(0)}-\rho \mathbf{u u u}-\theta \rho(\mathbf{u l}+$ cyclic $)$ is the difference between the third moment of the discrete equilibrium and the corresponding third moment (12) of the Maxwell-Boltzmann distribution.

The partial correction (14) described above leaves only the diagonal components of the $\Delta \mathbf{Q}^{(0)}$ tensor nonzero,

$$
\Delta Q_{x x x}^{(0)}=-\rho u_{x}^{3}, \quad \Delta Q_{y y y}^{(0)}=-\rho u_{y}^{3},
$$

while the off-diagonal terms $\Delta Q_{x x y}^{(0)}$ and $\Delta Q_{x y y}^{(0)}$ both vanish. The resulting viscous stress is thus [12]

$$
\boldsymbol{\Pi}^{(1)}=-\tau \rho \theta\left[(\nabla \mathbf{u})+(\nabla \mathbf{u})^{\top}\right]+\tau \partial_{x}\left(\rho u_{x}^{3}\right) \hat{\mathbf{x}} \hat{\mathbf{x}}+\tau \partial_{y}\left(\rho u_{y}^{3}\right) \hat{\mathbf{y}} \hat{\mathbf{y}}
$$

The off-diagonal component $\Pi_{x y}^{(1)}$ is correct, while the diagonal components $\Pi_{x x}^{(1)}$ and $\Pi_{y y}^{(1)}$ contain errors.

The $\Pi_{x x}^{(1)}$ component may be rewritten as

$$
\begin{aligned}
\Pi_{x x}^{(1)} & =-2 \tau \rho \theta \partial_{x} u_{x}+\tau \partial_{x}\left(\rho u_{x}^{3}\right), \\
& =-2 \tau \rho \theta \partial_{x} u_{x}+3 \tau \rho u_{x}^{2} \partial_{x} u_{x}+\tau u_{x}^{3} \partial_{x} \rho \\
& =-2 \tau \rho \theta\left[1-3 u_{x}^{2} /(2 \theta)\right] \partial_{x} u_{x}+\tau u_{x}^{3} \partial_{x} \rho .
\end{aligned}
$$

We now adjust the collision matrix $\Omega$ to apply a relaxation time $\tau_{x x}=\tau\left[1-3 u_{x}^{2} /(2 \theta)\right]^{-1}$ to $\Pi_{x x}$. Replacing the previous $\tau$ by $\tau_{x x}$ in the last line of (22) gives

$$
\Pi_{x x}^{(1)}=-2 \tau \rho \theta \partial_{x} u_{x}+\tau\left[1-3 u_{x}^{2} /(2 \theta)\right]^{-1} u_{x}^{3} \partial_{x} \rho
$$


Density fluctuations scale as $O\left(\mathrm{Ma}^{2}\right)$ at small Mach numbers, while the fluid velocity component $u_{x}$ is $O$ (Ma) in the conventional lattice units with $\xi_{i \alpha} \in\{-1,0,1\}$. The second term in (23) is therefore $O\left(\mathrm{Ma}^{4}\right)$ smaller than the first term, whereas the second term in (22) is only $O\left(\mathrm{Ma}^{2}\right)$ smaller than the first, since $u_{x}^{2} / \theta=O\left(\mathrm{Ma}^{2}\right)$. The error in $\Pi_{y y}^{(1)}$ may similarly be reduced by $O\left(\mathrm{Ma}^{2}\right)$ by taking the collision time for $\Pi_{y y}$ to be $\tau_{y y}=\tau\left[1-3 u_{y}^{2} /(2 \theta)\right]^{-1}$.

The presence of this remaining error due to density gradients suggests an advantage for the pseudo-incompressible lattice Boltzmann equilibria that replace the actual density $\rho$ by a reference density $\rho_{0}$ in all terms involving the fluid velocity [34]. The resulting hydrodynamic equations then yield the exact incompressibility condition $\nabla \cdot \mathbf{u}=0$ for steady flows, but otherwise propagate sound waves in almost the same way as the isothermal compressible Navier-Stokes equations. However, as shown in the appendix, the defect in Galilean invariance arising from these equilibria cannot be expressed as a divergence analogous to $\tau \nabla \cdot(\rho \mathbf{u u u})$, and therefore provides a much less promising starting point for a fully Galilean invariant algorithm.

\section{Implementation}

Further discretising the discrete Boltzmann equation (4) in space and time leads to the lattice Boltzmann equation

$$
\bar{f}_{i}\left(\mathbf{x}+\boldsymbol{\xi}_{i} \Delta t, t+\Delta t\right)=\bar{f}_{i}(\mathbf{x}, t)-\Delta t \sum_{j=0}^{N-1} \widetilde{\Omega}_{i j}\left(\bar{f}_{j}(\mathbf{x}, t)-f_{j}^{(0)}(\mathbf{x}, t)\right),
$$

for the transformed distribution functions

$$
\bar{f}_{i}(\mathbf{x}, t)=f_{i}(\mathbf{x}, t)+\frac{1}{2} \Delta t \sum_{j=0}^{N-1} \widetilde{\Omega}_{i j}\left(f_{j}(\mathbf{x}, t)-f_{j}^{(0)}(\mathbf{x}, t)\right),
$$

and the discrete collision matrix $\widetilde{\boldsymbol{\Omega}}=\left(\mathbf{I}+\frac{1}{2} \Delta t \boldsymbol{\Omega}\right)^{-1} \boldsymbol{\Omega}[29,35]$. These expressions reduce to those derived by He et al. [36] for the single-relaxation-time collision operator $\Omega=\tau^{-1} \mathbf{I}$. A recent rederivation of these results using Strang [37] splitting shows that the collision matrix $\boldsymbol{\Omega}$ may depend arbitrarily on the conserved moments $\rho$ and $\mathbf{u}$ without compromising second-order accuracy [35]. We use an overbar, as in $\overline{\boldsymbol{\Pi}}$, to indicate moments of the $\bar{f}_{i}$.

A convenient implementation takes any existing collision matrix with a single relaxation time $\tau$ for $\overline{\boldsymbol{\Pi}}$, and adjusts the collision times for the diagonal components of $\overline{\boldsymbol{\Pi}}$. For example, the post-collisional distribution functions on the D2Q9 lattice may be calculated as

$$
\begin{aligned}
\bar{f}_{i}^{\star}=\bar{f}_{i} & -\Delta t \sum_{j=0}^{8} \widetilde{\Omega}_{i j}\left(\bar{f}_{j}-f_{j}^{(0)}\right) \\
& -\frac{9}{2} w_{i}\left(\xi_{i x}^{2}-\frac{1}{3}\right)\left[\frac{\Delta t}{\tau\left(1-9 u_{x}^{2} / 2\right)+\Delta t / 2}-\frac{\Delta t}{\tau+\Delta t / 2}\right]\left(\bar{\Pi}_{x x}-\bar{\Pi}_{x x}^{(0)}\right) \\
& -\frac{9}{2} w_{i}\left(\xi_{i y}^{2}-\frac{1}{3}\right)\left[\frac{\Delta t}{\tau\left(1-9 u_{y}^{2} / 2\right)+\Delta t / 2}-\frac{\Delta t}{\tau+\Delta t / 2}\right]\left(\bar{\Pi}_{y y}-\bar{\Pi}_{y y}^{(0)}\right),
\end{aligned}
$$

where all distribution functions are evaluated at $(\mathbf{x}, t)$, and $\bar{\Pi}_{x x}$ and $\bar{\Pi}_{y y}$ are components of the pre-collisional second moment.

We have set $\theta=1 / 3$ in the formulae for $\tau_{x x}$ and $\tau_{y y}$ from the previous section. For sufficiently small Mach numbers, $|\mathbf{u}| \ll 1$ in lattice units, the two nonconstant divisors may be expanded for small $u_{x}$ and $u_{y}$ to obtain

$$
\begin{aligned}
\bar{f}_{i}^{\star}=\bar{f}_{i} & -\Delta t \sum_{j=0}^{8} \widetilde{\Omega}_{i j}\left(\bar{f}_{j}-f_{j}^{(0)}\right) \\
& \quad-\frac{36 \tau \Delta t}{4(\tau+\Delta t / 2)^{2}} w_{i}\left[\left(\xi_{i x}^{2}-\frac{1}{3}\right) u_{x}^{2}\left(\bar{\Pi}_{x x}-\bar{\Pi}_{x x}^{(0)}\right)+\left(\xi_{i y}^{2}-\frac{1}{3}\right) u_{y}^{2}\left(\bar{\Pi}_{y y}-\bar{\Pi}_{y y}^{(0)}\right)\right] .
\end{aligned}
$$

The additional $O\left(\mathrm{Ma}^{5}\right)$ error introduced by this approximation is the same order as the $u_{x}^{3} \partial_{x} \rho$ term in (23).

\section{Linear shear flows at arbitrary angles}

Measuring the decay rate of axis-aligned shear flows such as $\mathbf{u}=u(x, t) \hat{\mathbf{y}}$ superimposed on a uniform background velocity $\mathbf{U}$ is the common benchmark to test loss of Galilean invariance [6, 12, 23, 24]. However, this benchmark only tests the correctness of the off-diagonal component $\Pi_{x y}^{(1)}$ of the viscous momentum flux. We test the correctness of the full viscous momentum flux tensor using shear flows of the form

$$
\mathbf{u}=\mathbf{U}+\epsilon \hat{\mathbf{z}} \times \nabla \psi
$$

The small $O(\epsilon)$ deviations to the uniform background velocity $\mathbf{U}$ are obtained from the streamfunction

$$
\psi(x, y, t)=f(t) \sin (\mathbf{m} \cdot \mathbf{x}),
$$



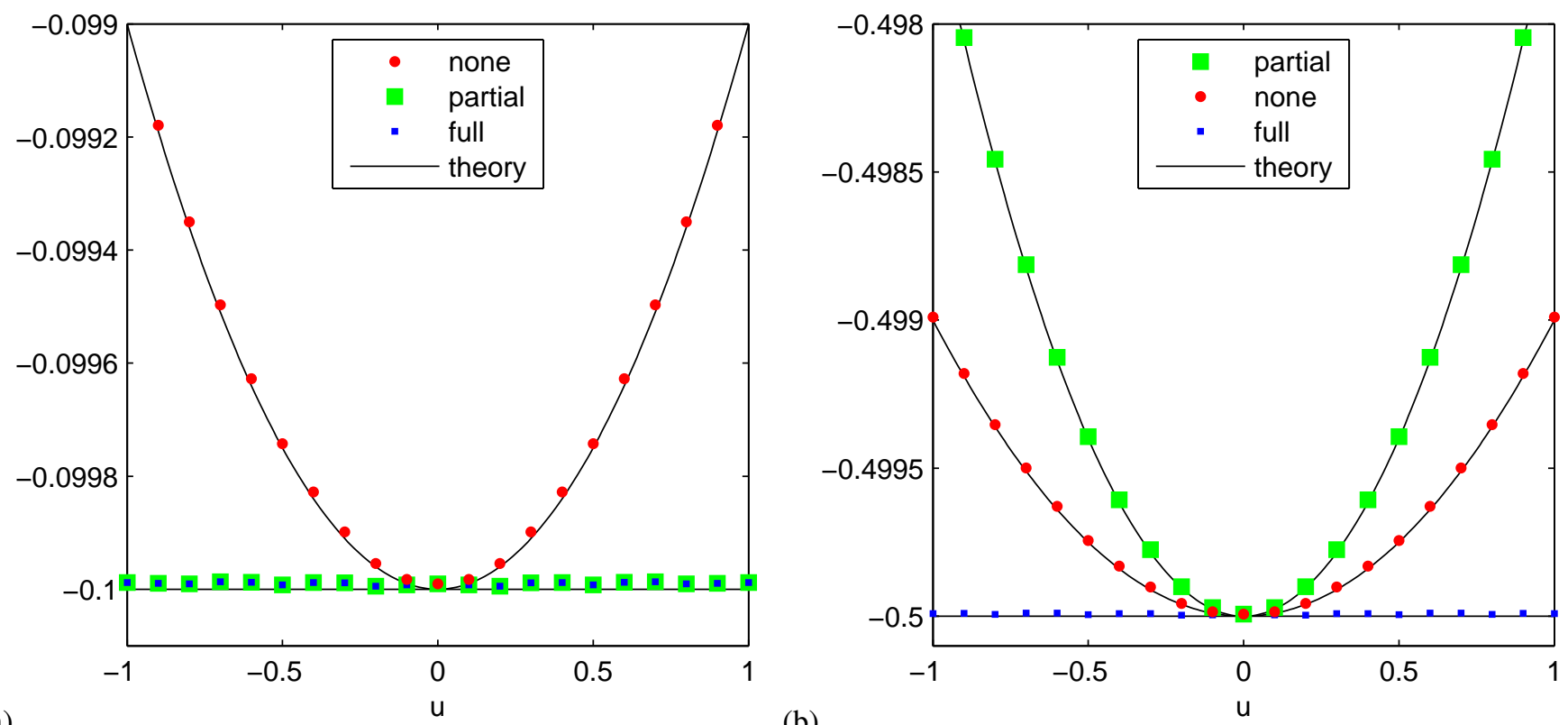

(a)

(b)

Figure 1: (a) Decay rate of axis-aligned sinusoidal shear flow $v=0.1$ and $\mathrm{Ma}=0.1$. The decay rate in the uncorrected scheme shows the theoretically expected proportionality to $1-\mathrm{Ma}^{2} u^{2}$, while the others are independent of $u$. (b) Decay rate for inclined sinusoidal shear with $\mathbf{m}=(1,2)$. The partially corrected scheme now also shows a $u$-dependence of the decay rate, and one which is larger than for the uncorrected scheme. The decay rate for the fully corrected scheme appears independent of $u$.

with $\mathbf{m}=(m, n)$ in the doubly-periodic domain $0 \leq x, y<2 \pi$. The small parameter $\epsilon$ is introduced to allow the nonlinear cubic error to be linearised around the background velocity $\mathbf{U}$. The streamfunction and the velocity components

$$
u_{x}=U_{x}-\epsilon n f(t) \cos (\mathbf{m} \cdot \mathbf{x}), \quad u_{y}=U_{y}+\epsilon m f(t) \cos (\mathbf{m} \cdot \mathbf{x}),
$$

are all periodic when $n$ and $m$ are integers. The standard axis-aligned shear flow tests correspond to $n=0$ or $m=0$. The theoretical decay due to a kinematic viscosity $v$ gives $f(t)=f(0) \exp \left(-v|\mathbf{m}|^{2} t\right)$ in the absence of cubic error terms.

Figure 1(a) shows the measured decay rate for axis-aligned shear flow with $m=1, n=0, v=0.1, \mathrm{Ma}=0.1$, and $\mathbf{U}=(0, U)$. All simulations were performed on $1024 \times 1024$ grids with periodic boundary conditions. This relatively high resolution ensures visual accuracy of the computed points in the figures with respect to spatial and temporal truncation errors. The uncorrected D2Q9 model shows the expected variation of the decay rate with the background velocity, the effective viscosity being proportional to $1-\mathrm{Ma}^{2} U^{2}$, while neither corrected model shows such a variation.

Figure 1(b) shows the corresponding results for a shear flow with $\mathbf{m}=(1,2)$ that is inclined relative to the coordinate axes, and a background flow $\mathbf{U}=(U, 0)$. Only the further corrected model with the $\mathbf{u}$-dependent collision operator exhibits Galilean-invariant behaviour. The partially corrected model with the cubic terms restored to $Q_{x x y}^{(0)}$ and $Q_{x y y}^{(0)}$ shows $U$-dependent variations in the decay rate that are even larger than those in the uncorrected model. This is due to the uncorrected diagonal components $\Pi_{x x}^{(1)}$ and $\Pi_{y y}^{(1)}$ of the viscous momentum flux.

To calculate the error in the partially corrected algorithm, it is convenient to eliminate the pressure by considering the vorticity equation

$$
\partial_{t} \omega+\mathbf{u} \cdot \nabla \omega=v \nabla^{2} \omega-\rho^{-1} \hat{\mathbf{z}} \cdot \nabla \times \nabla \cdot\left(\delta \mathbf{\Pi}^{(1)}\right)
$$

where $\omega=\hat{\mathbf{z}} \cdot \nabla \times \mathbf{u}=\nabla^{2} \psi$ with the sign convention in (28), and $\delta \boldsymbol{\Pi}^{(1)}=\tau \partial_{x}\left(\rho u_{x}^{3}\right) \hat{\mathbf{x}} \hat{\mathbf{x}}+\tau \partial_{y}\left(\rho u_{y}^{3}\right) \hat{\mathbf{y}} \hat{\mathbf{y}}$ is the non-Galilean-invariant error in the viscous stress given by (21). We neglect variations in density to obtain

$$
\partial_{t} \omega+\mathbf{u} \cdot \nabla \omega=v \nabla^{2} \omega+\tau \partial_{x y}\left[\partial_{x}\left(u_{x}^{3}\right)-\partial_{y}\left(u_{y}^{3}\right)\right] .
$$

For the small perturbations to a uniform flow $\mathbf{U}=\left(U_{x}, U_{y}\right)$ given by (28), we calculate

$$
\partial_{x}\left(u_{x}^{3}\right)=\partial_{x}\left[\left(U_{x}-\epsilon \psi_{y}\right)^{3}\right]=-3 \epsilon U_{x}^{2} \psi_{x y}+O\left(\epsilon^{2}\right),
$$

and similarly for $\partial_{y}\left(u_{y}^{3}\right)$, giving

$$
\partial_{t} \omega+\mathbf{u} \cdot \nabla \omega=v \nabla^{2} \omega-3 \epsilon \tau|\mathbf{U}|^{2} \psi_{x x y y} .
$$

The decay rate for flows of the form (29) is thus

$$
\sigma=-v\left(|\mathbf{m}|^{2}-3 \mathrm{Ma}^{2}|\mathbf{U}|^{2} m^{2} n^{2} /|\mathbf{m}|^{2}\right) .
$$

The error in $\sigma$ is proportional to the product $n m$, which explains why no error appears in axis-aligned flows with $m=0$ or $n=0$. Moreover, the error is independent of the orientation of the background $\mathbf{U}$ relative to the vector $\mathbf{m}$. Due to the factor of 3 , the error term may be larger in magnitude than the corresponding term $\mathrm{Ma}^{2}|\mathbf{U} \cdot \mathbf{m}|^{2}$ for the uncorrected algorithm, as demonstrated for the parameters $\mathbf{m}=(1,2)$ in Fig. $1(b)$. 


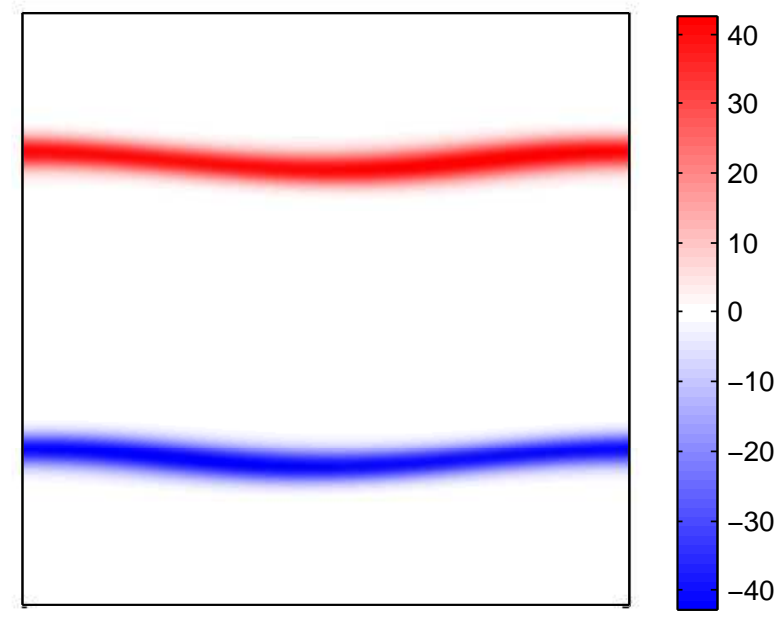

(a)

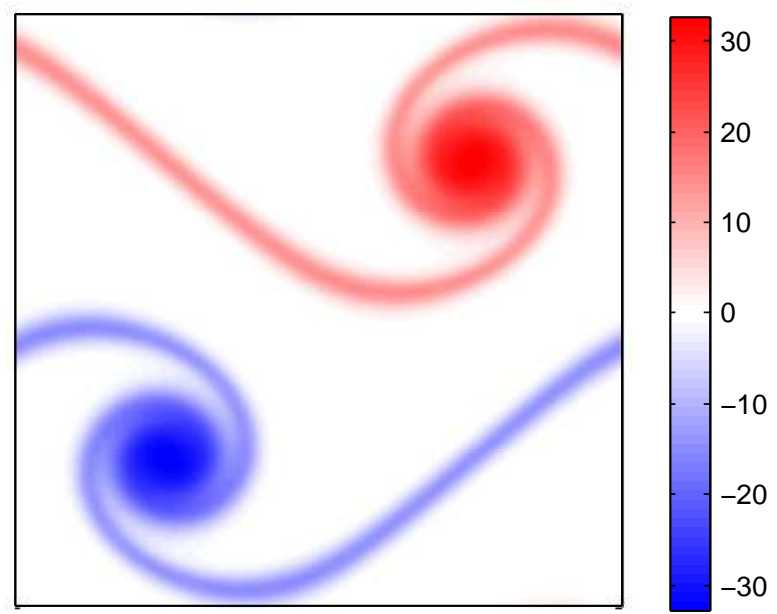

(b)

Figure 2: The rolled up vorticity in the perturbed anti-parallel shear layers at (a) $t=0.25$ and (b) $t=1.0$ for the Minion and Brown [38] initial conditions.

\section{Nonlinear simulations}

For the more demanding test of an evolving flow with density variations we employ the initial conditions used by Minion and Brown [38]

$$
u_{x}=\left\{\begin{array}{ll}
\tanh (\kappa(y-1 / 4)), & y \leq 1 / 2, \\
\tanh (\kappa(3 / 4-y)), & y>1 / 2,
\end{array} \quad u_{y}=\delta \sin (2 \pi(x+1 / 4))\right.
$$

with parameters $\kappa=80, \delta=0.05$ in the doubly-periodic domain $0 \leq x, y<1$. The sinusoidal perturbation $u_{y}$ triggers a Kelvin-Helmholtz instability in the two anti-parallel shear layers, which subsequently roll up into spiral vortices as shown in Fig. 2

In the absence of cubic and other errors, the lattice Boltzmann algorithm yields solutions of the two-dimensional isothermal compressible Navier-Stokes equations in the form

$$
\partial_{t} \rho+\nabla \cdot(\rho \mathbf{u})=0, \quad \partial_{t} \mathbf{u}+\mathbf{u} \cdot \nabla \mathbf{u}+c_{\mathrm{s}}^{2} \rho^{-1} \nabla \rho=\rho^{-1} \nabla \cdot\left\{\rho v\left[(\nabla \mathbf{u})+(\nabla \mathbf{u})^{\top}\right]\right\},
$$

where $c_{\mathrm{s}}^{2}=\theta$ is the sound speed squared. A constant collision time $\tau$ implies a constant kinematic viscosity $v$, and hence a dynamic viscosity $\mu=\rho v$ proportional to the local density. A lattice Boltzmann solution may differ from the exact solution of the PDE system (37) through three different sources of error. There is the error due to the $\nabla \cdot(\rho \mathbf{u u u})$ term in the viscous stress or its various partially corrected forms, there is the space/time truncation error that should be $O\left(n^{-2}\right)$ on a lattice with $n \times n$ points, and finally there is an error due to the $\boldsymbol{\Pi}^{(2)}$ and higher terms in the Chapman-Enskog expansion (8). These so-called Burnett terms cause solutions of the discrete Boltzmann PDE system (4) to differ by $O\left(K n^{2}\right)$ from solutions of the Navier-Stokes equations (37).

Figure 3 shows the behaviour of the discrete $\ell_{2}$-norm errors in the vorticity field at $t=1$ of the various lattice Boltzmann models with $\mathrm{Re}=2000$ and $\mathrm{Ma}=128 \sqrt{3} / 2217 \approx 0.100001$ relative to a reference solution. This choice of Mach number ensures that $t=1$ is reached exactly after an integer number of timesteps when $n=512$ or a higher power of 2 . A highly accurate reference solution to (37) was computed using a Fourier pseudo-spectral discretisation in space and fourth-order Runge-Kutta integration in time of the resulting system of ordinary differential equations. This explicit treatment of the viscous term implies a stringent limit $\Delta t \leq 2.7 \mathrm{Re} / k_{\max }^{2}$ on the maximum stable timestep $\Delta t$, where $k_{\max }=2^{1 / 2} \pi M$ for a simulation with $M \times M$ Fourier collocation points. The solution was converged for $M=512$.

The total errors in the solutions computed using the standard and partially corrected lattice Boltzmann algorithm saturate at around $n=1024$, and do not decrease under further grid refinement to $n=2048$ and $n=4096$. By contrast, the total error in the solution computed using the fully corrected lattice Boltzmann algorithm remains consistent with second-order convergence. However, this is only because the Burnett error, and the remaining $O\left(\mathrm{Ma}^{5}\right)$ error in the viscous stress, are not significant compared with the truncation errors for this Mach number, Reynolds number, and maximum resolution. These other sources of error are, though, the most likely explanation for the deviation from second-order convergence at $n=4096$.

Also shown in Fig. 3 are the errors in solutions computed using a 2Q13 lattice Boltzmann model with the discrete equilibria from Weimar and Boon [9]

$$
f_{i}^{(0)}=\rho\left\{W_{i}\left[1+2 \mathbf{u} \cdot \boldsymbol{\xi}_{i}+2\left(\mathbf{u} \cdot \boldsymbol{\xi}_{i}\right)^{2}-|\mathbf{u}|^{2}\right]+E_{i}\left(\mathbf{u} \cdot \boldsymbol{\xi}_{i}\right)^{3}-F_{i}\left(\mathbf{u} \cdot \boldsymbol{\xi}_{i}\right)|\mathbf{u}|^{2}\right\},
$$




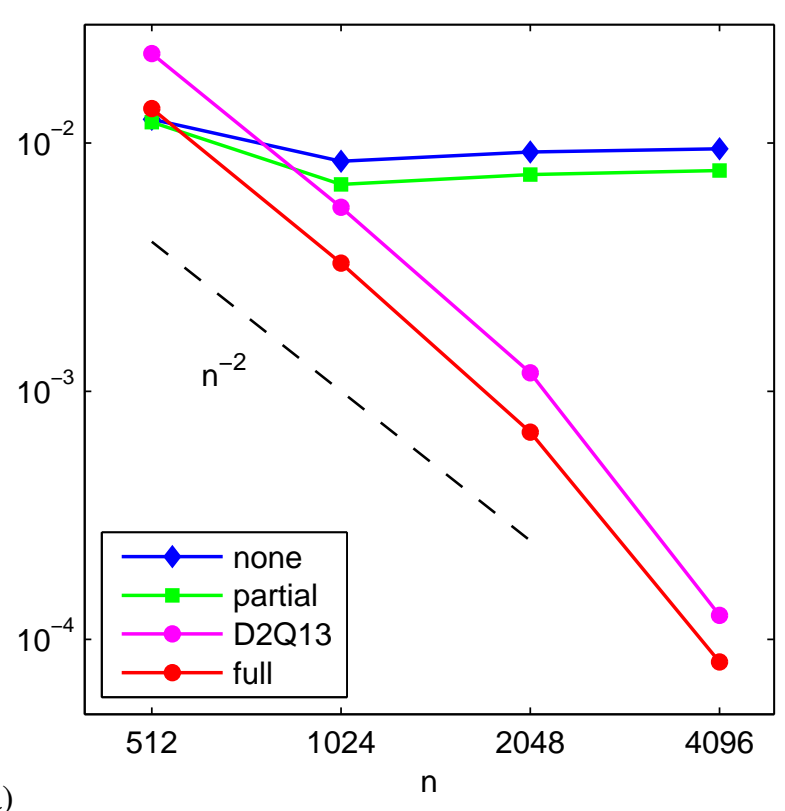

(a)

Figure 3: Errors in (a) vorticity and (b) density at $t=1$ for $\mathrm{Re}=2000$ and Ma $=0.1$ on successively finer $n \times n$ grids. The errors in the standard and partially corrected D2Q9 simulations soon become dominated by the $O\left(\mathrm{Ma}^{3}\right)$ error rather than the space/time discretisation error, and show no improvement under further grid refinement. By contrast, the fully corrected D2Q9 simulation is more accurate than the D2Q13 simulation, which has the correct $\mathbf{Q}^{(0)}$ tensor and no $\nabla \cdot(\rho \mathbf{u u u})$ error. The errors run from largest (top) to smallest (bottom) in the figure legends.

with the weights

$$
W_{i}=\left\{\begin{array}{l}
36 / 96, \\
8 / 96, \\
6 / 96, \\
1 / 96,
\end{array} \quad E_{i}=\left\{\begin{array}{l}
0, \\
32 / 96, \\
12 / 96, \\
1 / 96,
\end{array} \quad F_{i}= \begin{cases}0, & \text { for } i=0, \\
48 / 96, & \text { for } i=1,2,3,4, \\
12 / 96, & \text { for } i=5,6,7,8, \\
0, & \text { for } i=9,10,11,12 .\end{cases}\right.\right.
$$

The additional 4 axis-aligned particle velocities with $\left|\boldsymbol{\xi}_{i}\right|=2$ enable all four components of the $\mathbf{Q}^{(0)}$ tensor to be set to their Maxwell-Boltzmann values as in (12). This completely removes the $\nabla \cdot(\rho \mathbf{u u u})$ term from the simulated viscous stress. The Weimar-Boon model gives the different equation of state $c_{\mathrm{s}}^{2}=1 / 2$ in lattice units, so it was run with $\mathrm{Ma}=128 \sqrt{2} / 1810 \approx$ 0.100011. Again, this Mach number was chosen so that $t=1$ was reached exactly after an integer number of timesteps. The data in Fig. 3 show the errors relative to a second converged spectral simulation with this slightly different Mach number. The fully corrected D2Q9 solution is thus more accurate than the D2Q13 solution, even though the latter exactly reproduces the Navier-Stokes viscous stress, while the former retains an $O\left(\mathrm{Ma}^{5}\right)$ error.

\section{Three dimensions}

Keating et al. [24] investigated partial cubic corrections in three dimensions using the equilibria

$$
f_{i}^{(0)}=\rho w_{i}\left\{1+3 \mathbf{u} \cdot \boldsymbol{\xi}_{i}+\frac{9}{2}\left(\mathbf{u} \cdot \boldsymbol{\xi}_{i}\right)^{2}-\frac{3}{2}|\mathbf{u}|^{2}+\frac{9}{2}\left(\mathbf{u} \cdot \boldsymbol{\xi}_{i}\right)\left[\left(\mathbf{u} \cdot \boldsymbol{\xi}_{i}\right)^{2}-|\mathbf{u}|^{2}\right]\right\} .
$$

These equilibria give correct values for the off-diagonal components such as $Q_{x x y}^{(0)}$ on the D3Q27 lattice with the weights given in Table 1. However, contrary to the assertion of Keating et al. [24], these equilibria give incorrect off-diagonal components when used with the D3Q15 or D3Q19 lattices. A direct evaluation using velocities and weights from Table 1 gives

$$
Q_{x x y}^{(0)}=\frac{1}{3} \rho u_{y}+\rho\left(u_{x}^{2}+u_{z}^{2}\right) u_{y}, \quad Q_{x y z}^{(0)}=3 \rho u_{x} u_{y} u_{z}
$$

for the D3Q15 lattice, and

$$
Q_{x x y}^{(0)}=\frac{1}{3} \rho u_{y}+\rho\left(u_{x}^{2}-u_{z}^{2} / 2\right) u_{y}, \quad Q_{x y z}^{(0)}=0,
$$

for the D3Q19 lattice. In particular, $Q_{x y z}^{(0)}$ is identically zero for the D3Q19 lattice because no particle has all three of its velocity components non-zero. The only elements of the orthogonal bases for the D3Q19 lattice that are cubic in $\xi_{i}$ are the vector $\left(\left|\xi_{i}\right|^{2}-\alpha\right) \xi_{i}$, and the three quantities $\left(\xi_{i y}^{2}-\xi_{i z}^{2}\right) \xi_{i x}$ plus cyclic permutations [28, 39]. The constant $\alpha$ in the vector is $\alpha=9 / 5$ for an $\ell_{2}$-orthogonal basis, and $\alpha=5 / 3$ for a $w_{i}$-orthogonal basis. None of these basis vectors project onto $\xi_{i x} \xi_{i y} \xi_{i z}$.

It is thus impossible to correct the off-diagonal entries of $\mathbf{Q}^{(0)}$ on the D3Q15 and D3Q19 lattices, but these smaller lattices have other undesirable properties. Simulations using the D3Q15 lattice are susceptible to checkerboard artifacts [40, 41] because 


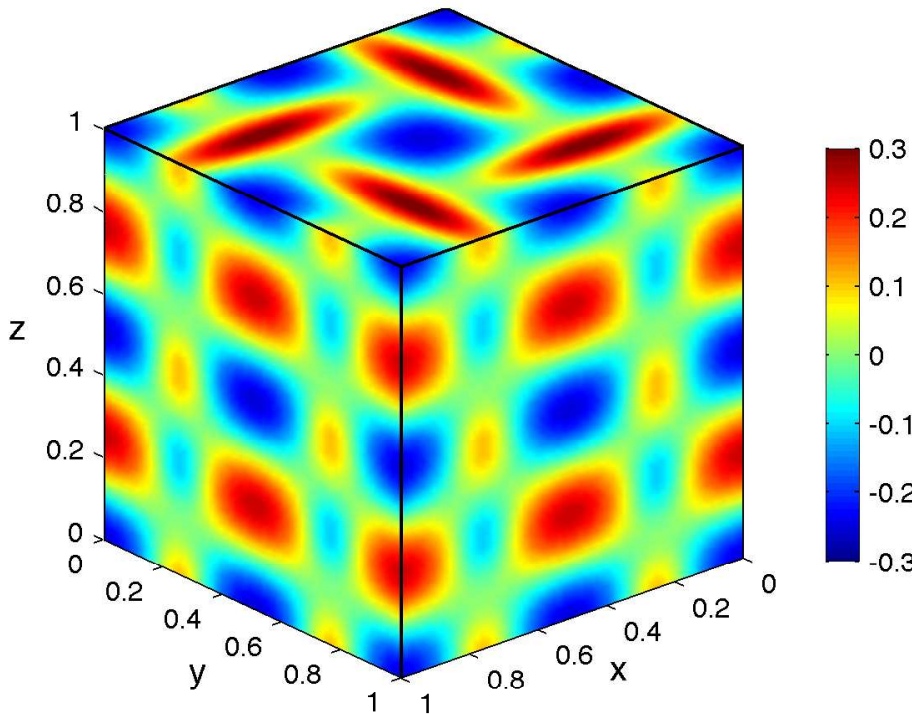

(a)

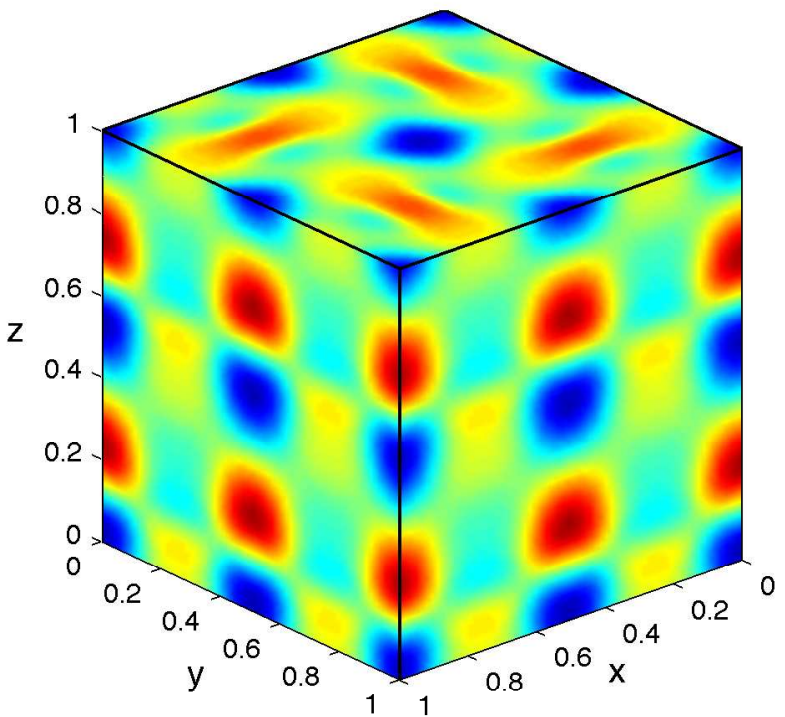

(b)

Figure 4: Development of the vertical velocity $w$ from planar initial conditions in three-dimensional Taylor-Green flow with $U=0$. Plot (a) shows data at $t=3$ for $\operatorname{Re}=30$, and plot (b) shows data at $t=10$ for $\operatorname{Re}=100$ on the same colour scale.

this lattice decouples into two sublattices that are distinguished by $x+y+z$ being odd or even on alternate timesteps [28]. More recently, growing evidence has emerged of artifacts caused by insufficient isotropy in simulations using the previously popular D3Q19 lattice. These artifacts are especially visible in flows that should be axisymmetric, and are greatly reduced by using the D3Q27 lattice [42-45].

Having corrected the off-diagonal elements of $\mathbf{Q}^{(0)}$ using the equilibria (40) on the D3Q27 lattice, the earlier formula (26) for correcting the remaining defects in the post-collisional distribution functions extends into three dimensions as

$$
\begin{aligned}
\bar{f}_{i}^{\star}=\bar{f}_{i} & -\Delta t \sum_{j=0}^{N-1} \widetilde{\Omega}_{i j}\left(\bar{f}_{j}-f_{j}^{(0)}\right) \\
& -\sum_{\alpha \in\{x, y, z\}} \frac{9}{2} w_{i}\left(\xi_{i \alpha}^{2}-\frac{1}{3}\right)\left(\frac{\Delta t}{\tau\left(1-9 u_{\alpha}^{2} / 2\right)+\Delta t / 2}-\frac{\Delta t}{\tau+\Delta t / 2}\right)\left(\Pi_{\alpha \alpha}-\Pi_{\alpha \alpha}^{(0)}\right),
\end{aligned}
$$

where the sum is taken over the three coordinates $x, y, z$. Again, some simplification is available by expanding the denominators for $|\mathbf{u}| \ll 1$ to obtain the three-dimensional analogue of (27),

$$
\bar{f}_{i}^{\star}=\bar{f}_{i}-\Delta t \sum_{j=0}^{N-1} \widetilde{\Omega}_{i j}\left(\bar{f}_{j}-f_{j}^{(0)}\right)-\frac{36 \tau \Delta t}{4(\tau+\Delta t / 2)^{2}} \sum_{\alpha \in\{x, y, z\}} w_{i}\left(\xi_{i \alpha}^{2}-\frac{1}{3}\right) u_{\alpha}^{2}\left(\Pi_{\alpha \alpha}-\Pi_{\alpha \alpha}^{(0)}\right) .
$$

We evaluate this correction using simulations of the three-dimensional Taylor-Green [46] vortex studied by Orszag [47]. Adding a uniform background velocity $\mathbf{U}=(U, U, U)$ to the initial conditions gives

$$
u_{x}=\cos x \sin y \cos z+U, \quad u_{y}=-\sin x \cos y \cos z+U, \quad u_{z}=U,
$$

in the triply-periodic domain $0 \leq x, y, z<2 \pi$. These initial conditions are divergence-free, and evolve into a fully threedimensional flow for $t>0$. The resulting vertical velocity components for $\operatorname{Re}=30$ and $\operatorname{Re}=100$ are shown in Fig. 4 . The initial conditions were translated by $\pi / 4$ in the $z$ direction so that $\cos (z+\pi / 4)$ appeared in the initial conditions. Otherwise the vertical velocity on $z=2 \pi$ vanishes due to the symmetry of the initial conditions.

Using $\mathbf{u}_{0}(\mathbf{x}, t)$ to denote the solution evolving from the initial conditions with $U=0$, Galilean invariance implies

$$
\mathbf{u}(\mathbf{x}, t)=\mathbf{u}_{0}(\mathbf{x}-t \mathbf{U}, t)+\mathbf{U},
$$

where the difference $\mathbf{x}-t \mathbf{U}$ is computed modulo $2 \pi$ in each component. We measure the extent to which the various lattice Boltzmann algorithms deviate from exact Galilean invariance by computing the difference between two lattice Boltzmann solutions $\tilde{\mathbf{u}}(\mathbf{x}, t)$ and $\tilde{\mathbf{u}}_{0}(\mathbf{x}, t)$, the latter computed with $U=0$, using

$$
\mathcal{E}(U, t)=\left(n^{-3} \sum_{k}\left|\tilde{\mathbf{u}}\left(\mathbf{x}_{k}, t\right)-\mathbf{U}-\tilde{\mathbf{u}}_{0}\left(\mathbf{x}_{k}-t \mathbf{U}, t\right)\right|^{2}\right)^{1 / 2} .
$$




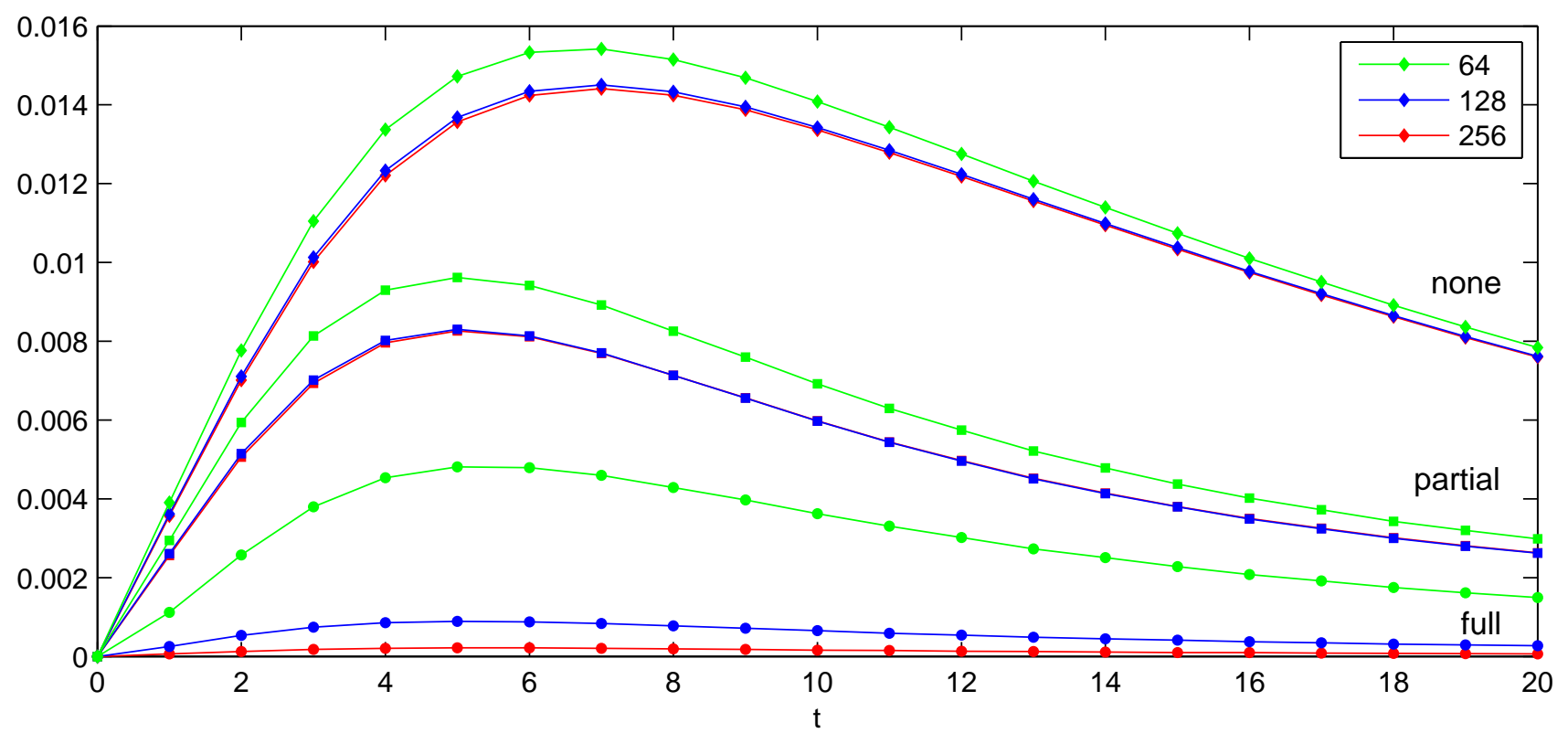

Figure 5: Defects in Galilean invariance for $\mathrm{Re}=30$ and $\mathrm{Ma}=\sqrt{3} /(4 \pi) \approx 0.14$ on $64^{3}, 128^{3}$ and $256^{3}$ lattices with no, partial, or full correction of the cubic error term. The errors in the first two sets of simulations become dominated by the cubic errors at the $128^{3}$ resolution, so refining to $256^{3}$ gives no visible improvement. The errors in the final set of simulations continue to decrease with grid refinement.

The sum is take over all $n^{3}$ grid points $\mathbf{x}_{k}$, and the difference $\mathbf{x}_{k}-t \mathbf{U}$ is again computed modulo $2 \pi$.

Figure 5 shows $\mathcal{E}(U, t)$ as a function of $t$ for $U=1 / 4, \operatorname{Re}=30, \mathrm{Ma}=\sqrt{3} /(4 \pi) \approx 0.14$ for the three different algorithms on grids with $n=64,128,256$. The differences for the algorithms with no or partial correction of the cubic error terms are virtually identical between the $128^{3}$ and $256^{3}$ simulations, showing that the defect in Galilean invariance is dominated by the cubic error term rather than by truncation error. By contrast, the defect in Galilean invariance for the fully corrected algorithm decreases by about a factor of 4 between the $128^{3}$ and $256^{3}$ simulations, consistent with the remaining defect being primarily due to truncation error. The $O\left(\mathrm{Ma}^{5}\right)$ error due to the uncorrected density gradient is not visible for these parameters. Figure 6 shows the same data for simulations with $\mathrm{Re}=100$, but all other parameters unchanged. The standard lattice Boltzmann algorithm becomes unstable for these parameters, while the defect in Galilean invariance for the partially corrected algorithm again saturates at a finite value with increasing resolution.

\section{Further correction with adjustable bulk viscosity}

The more general form of the Navier-Stokes viscous stress is

$$
\mathbf{\Pi}^{(1)}=-\mu\left[(\nabla \mathbf{u})+(\nabla \mathbf{u})^{\top}\right]-\lambda(\nabla \cdot \mathbf{u}) \mathbf{I}
$$

The $\lambda$ term adds an additional bulk viscosity that enhances the dissipation of sound waves. A bulk viscosity much larger than the shear viscosity is commonly used to improve the stability of lattice Boltzmann algorithms in the weakly compressible regime $[27,28,48,49]$, but also enlarges the non-Galilean contribution to the viscous stress from the trace of the spurious $\nabla \cdot(\rho \mathbf{u u u})$ term.

The off-diagonal components of $\mathbf{\Pi}^{(1)}$ are unaffected by this change, so we consider just the diagonal components

$$
\Pi_{x x}^{(1)}=-(2 \mu+\lambda) \partial_{x} u_{x}-\lambda \partial_{y} u_{y}, \quad \Pi_{y y}^{(1)}=-(2 \mu+\lambda) \partial_{y} u_{y}-\lambda \partial_{x} u_{x},
$$

in two dimensions for simplicity. We write this pair of equations in matrix form as

$$
\left(\begin{array}{c}
\Pi_{x x}^{(1)} \\
\Pi_{y y}^{(1)}
\end{array}\right)=-\left(\begin{array}{cc}
\frac{\lambda+2 \mu}{1-9 u_{x}^{2} / 2} & \frac{\lambda}{1-9 u_{y}^{2} / 2} \\
\frac{\lambda}{1-9 u_{x}^{2} / 2} & \frac{\lambda+2 \mu}{1-9 u_{y}^{2} / 2}
\end{array}\right)\left(\begin{array}{l}
\left(1-9 u_{x}^{2} / 2\right) \partial_{x} u_{x} \\
\left(1-9 u_{y}^{2} / 2\right) \partial_{y} u_{y}
\end{array}\right),
$$

and invert to obtain the collision matrix

$$
\boldsymbol{\Omega}=-\left(\begin{array}{cc}
\frac{(\lambda+2 \mu)\left(2-9 u_{x}^{2}\right)}{8 \mu(\lambda+\mu)} & \frac{\lambda\left(2-9 u_{x}^{2}\right)}{8 \mu(\lambda+\mu)} \\
\frac{\lambda\left(2-9 u_{y}^{2}\right)}{8 \mu(\lambda+\mu)} & \frac{(\lambda+2 \mu)\left(2-9 u_{y}^{2}\right)}{8 \mu(\lambda+\mu)}
\end{array}\right)
$$




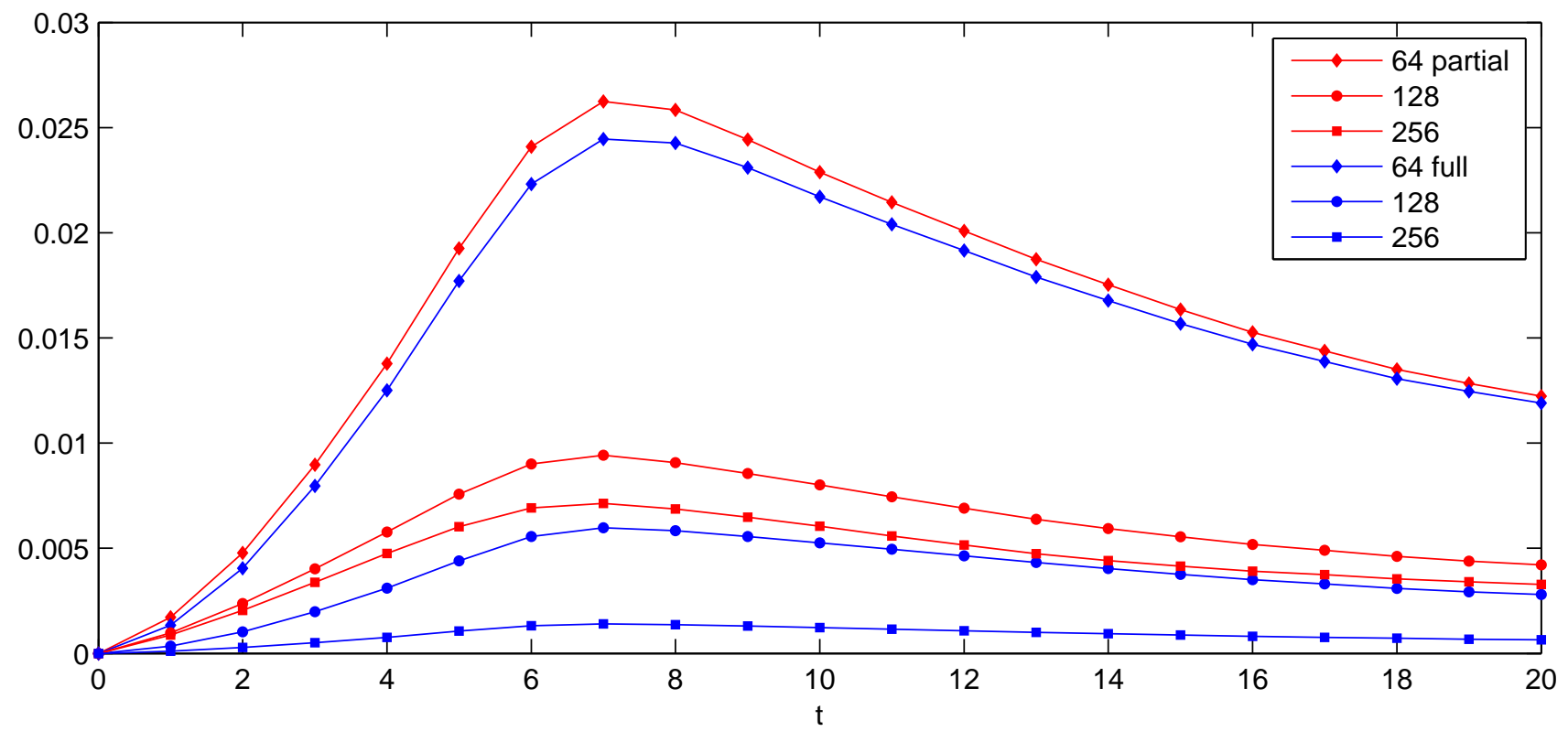

Figure 6: Defects in Galilean invariance for $\mathrm{Re}=100$ and $\mathrm{Ma}=\sqrt{3} /(4 \pi) \approx 0.14$ on $64^{3}, 128^{3}$ and $256^{3}$ lattices (top to bottom) with partial or full correction of the cubic error term. The defect in the fully corrected simulations appears to converge towards zero under grid refinement, while the defect in the partially corrected simulations appears to approach a non-zero value.

for the discrete Boltzmann PDE. The previous formula $\widetilde{\mathbf{\Omega}}=\left(\mathbf{I}+\frac{1}{2} \Delta t \mathbf{\Omega}\right)^{-1} \mathbf{\Omega}$ then gives the discrete collision matrix, with components such as

$$
\widetilde{\Omega}_{x x}=\frac{2 \Delta t\left[\Delta t\left(2-9 u_{y}^{2}\right)-4(\lambda+2 \mu)\right]\left(2-9 u_{x}^{2}\right)}{64 \mu(\lambda+\mu)-4 \Delta t(\lambda+2 \mu)\left(4-9 u_{x}^{2}-9 u_{y}^{2}\right)+\Delta t^{2}\left(2-9 u_{x}^{2}\right)\left(2-9 u_{y}^{2}\right)} .
$$

This approach extends easily to a $3 \times 3$ matrix for the three diagonal components $\Pi_{x x}^{(1)}, \Pi_{y y}^{(1)}, \Pi_{z z}^{(1)}$ in three dimensions.

\section{Conclusion}

The derivation of hydrodynamic equations from discrete kinetic theory via a system of moment equations follows the same route as the derivation from the continuous Boltzmann equation. However, most common lattice Boltzmann algorithms use quadratic polynomials in $\mathbf{u}$ for their equilibrium distributions. The third equilibrium moment $\mathbf{Q}^{(0)}$ therefore omits the cubic term $\rho \mathbf{u u u}$ that is present in the corresponding equilibrium moment for continuous kinetic theory. This leads to an erroneous term proportional to $\nabla \cdot(\rho \mathbf{u u u})$ in the viscous stress that violates Galilean invariance [6].

The missing cubic term is not easily restored because the discrete velocity components $\xi_{i \alpha}$ lie in the set $\{-1,0,1\}$ for common lattices such as D2Q9, D3Q15, D3Q19, and D3Q27. Since $\xi_{i \alpha}^{3}=\xi_{i \alpha}$, the diagonal components of $\mathbf{Q}^{(0)}$ are set by the first moment $\rho \mathbf{u}$ and cannot be varied independently. The most common remedy adopts a larger lattice, such as D2Q13 or D2Q17, with particles moving with $\left|\xi_{i}\right|=2$ or $\left|\xi_{i}\right|=2^{3 / 2}$. This remove the previous constraint, but adds computational cost and increases susceptibility to numerical instabilities at the grid scale. Another approach, primarily used in the free-energy approach to multiphase flow, adds finite difference terms to correct the momentum flux.

On the D2Q9 lattice the cubic terms may be restored to the off-diagonal components $Q_{x x y}^{(0)}$ and $Q_{x y y}^{(0)}$. This corrects the lack of Galilean invariance visible in axis-aligned shear flows [12, 23, 24], in which only the $\Pi_{x y}$ component of the momentum flux tensor plays a rôle. However, the diagonal components $\Pi_{x x}$ and $\Pi_{y y}$ remain incorrect, causing visible defects for shear flows in directions inclined to the axes of the discrete velocity lattice. For example, the $x x$ component of the viscous stress is

$$
\Pi_{x x}^{(1)}=-\tau_{x x}\left[(2 / 3) \rho \partial_{x} u_{x}-\partial_{x}\left(\rho u_{x}^{3}\right)\right]=-\tau_{x x}\left[(2 / 3) \rho\left(1-9 u_{x}^{2} / 2\right) \partial_{x} u_{x}+u_{x}^{3} \partial_{x} \rho\right]
$$

where $\tau_{x x}$ is the relaxation time applied to $\Pi_{x x}$ in the collision operator. This still contains the non-Galilean-invariant term $\partial_{x}\left(\rho u_{x}^{3}\right)$. Moreover, the partial correction of $\mathbf{Q}^{(0)}$ may increase the error for shear flows inclined to the underlying lattice, as shown in Fig. 1(b).

In this paper we adopted a matrix collision operator that applies different relaxation times to the different components of $\boldsymbol{\Pi}$. Setting the relaxation time for $\Pi_{x x}$ to be $\tau_{x x}=\tau /\left(1-9 u_{x}^{2} / 2\right)$ absorbs the first part of the defect in (53), leaving only a term proportional to $\partial_{x} \rho$. Similarly redefining $\tau_{y y}=\tau /\left(1-9 u_{y}^{2} / 2\right)$ absorbs the corresponding part of the defects in $\Pi_{y y}$. This completely eliminates the defects in Galilean invariance for flows with constant density, as demonstrated for twodimensonal linear shear flows inclined at arbitrary angles to the discrete velocity lattice. For more general flows, in which density fluctuations are non-zero but $O\left(\mathrm{Ma}^{2}\right)$, the defect in Galilean invariance is reduced from $O\left(\mathrm{Ma}^{3}\right)$ to $O\left(\mathrm{Ma}^{5}\right)$. Numerical comparisons with independent pseudospectral simulations of the two-dimensional isothermal Navier-Stokes equations confirm 
that a substantial error attributable to lack of Galilean invariance persists in the partially corrected algorithm, but is greatly reduced in magnitude in the further corrected D2Q9 lattice Boltzmann algorithm. The latter then becomes as accurate as the Weimar-Boon D2Q13 formulation that completely eliminates the cubic error term.

This approach extends to three dimensions, but only for the D3Q27 lattice that is a tensor product of one-dimensional three-velocity lattices in each coordinate. All components of $\mathbf{Q}^{(0)}$ other than the diagonal components $Q_{x x x}, Q_{y y y}, Q_{z z z}$ may be corrected by the addition of a cubic term to the equilibrium distributions. This partial correction of the off-diagonal components of $\mathbf{Q}^{(0)}$ is not possible for the smaller D3Q15 or D3Q19 lattices. However, the popularity of these lattices is already diminishing due to the presence of checkerboard artifacts on the D3Q15 lattice, and increasing evidence for loss of isotropy when using the D3Q19 lattice. The remaining defects in the diagonal components of $\boldsymbol{\Pi}$ on the D3Q27 lattice may be corrected by adjusting the collision times $\tau_{x x}, \tau_{y y}, \tau_{z z}$ through a straightforward modification of any existing collision operator with a uniform stress relaxation time. The resulting algorithm greatly reduces the defects due to lack of Galilean invariance in three-dimensional simulations of the Taylor-Green vortex with a superimposed uniform background flow. It retains the standard lattice Boltzmann streaming and collision steps without needing additional interpolation or calculation of finite difference approximations. The small additional complexity of the collision operator will therefore have a negligible effect upon the speed of simulations, a speed typically limited by memory bandwidth rather than by floating point computations.

\section{A. The viscous stress from pseudo-incompressible equilibria}

The pseudo-incompressible lattice Boltzmann equilibria [34]

$$
f_{i}^{(0)}=w_{i}\left[\rho+\rho_{0}\left(3 \mathbf{u} \cdot \boldsymbol{\xi}_{i}+\frac{9}{2}\left(\mathbf{u} \cdot \boldsymbol{\xi}_{i}\right)^{2}-\frac{3}{2}|\mathbf{u}|^{2}\right)\right],
$$

replace the density $\rho$ by a constant reference density $\rho_{0}$ in every term involving the fluid velocity. These equilibria thus differ by $O\left(\mathrm{Ma}^{3}\right)$ from the standard equilibria in (2)

The pseudo-incompressible equilibria give the fluid equations

$$
\partial_{t} \rho+\rho_{0} \nabla \cdot \mathbf{u}=0, \quad \rho_{0}\left(\partial_{t} \mathbf{u}+\mathbf{u} \cdot \nabla \mathbf{u}\right)+\theta \nabla \rho=-\nabla \cdot \mathbf{\Pi}^{(1)}
$$

which may be identified with Chorin's artificial compressibility equations [50]. The equilibria (54) are commonly called "incompressible" because $\nabla \cdot \mathbf{u}=0$ holds exactly for steady states. However, the above fluid equations still support sound waves with velocity $c_{\mathrm{s}}=\theta^{1 / 2}$, and the equilibria differ by only $O\left(\mathrm{Ma}^{3}\right)$ from the standard quadratic polynomial equilibria (2). Unsteady solutions to (55) therefore exhibit the same $O\left(\mathrm{Ma}^{2}\right)$ compressibility errors, relative to the incompressible limit, as solutions of the isothermal Navier-Stokes equations (37).

The leading order time derivation of $\mathbf{\Pi}^{(0)}=\rho \theta \mathbf{I}+\rho_{0} \mathbf{u} \mathbf{u}$ is

$$
\begin{aligned}
\partial_{t_{0}} \boldsymbol{\Pi}^{(0)} & =\mathbf{I} \theta\left(\partial_{t_{0}} \rho\right)+\rho_{0} \mathbf{u}\left(\partial_{t_{0}} \mathbf{u}\right)+\rho_{0}\left(\partial_{t_{0}} \mathbf{u}\right) \mathbf{u} \\
& =-\mathbf{I} \theta \rho_{0} \nabla \cdot \mathbf{u}-\rho_{0} \mathbf{u}\left(\mathbf{u} \cdot \nabla+\frac{\theta}{\rho_{0}} \nabla \rho\right)-\rho_{0}\left(\mathbf{u} \cdot \nabla+\frac{\theta}{\rho_{0}} \nabla \rho\right) \mathbf{u}
\end{aligned}
$$

The third moment of the equilibrium is $Q_{\alpha \beta \gamma}^{(0)}=\rho_{0} \theta\left(u_{\alpha} \delta_{\beta \gamma}+u_{\beta} \delta_{\gamma \alpha}+u_{\gamma} \delta_{\alpha \beta}\right)$, with divergence

$$
\nabla \cdot \mathbf{Q}^{(0)}=\rho_{0} \theta\left[(\nabla \mathbf{u})+(\nabla \mathbf{u})^{\top}+I \nabla \cdot \mathbf{u}\right] .
$$

Combining these two gives a viscous stress proportional to

$$
\partial_{t_{0}} \boldsymbol{\Pi}^{(0)}+\nabla \cdot \mathbf{Q}^{(0)}=\theta \rho_{0}\left[(\nabla \mathbf{u})+(\nabla \mathbf{u})^{\top}\right]-\theta[\mathbf{u}(\nabla \rho)+(\nabla \rho) \mathbf{u}]-\rho_{0}[\mathbf{u}(\mathbf{u} \cdot \nabla \mathbf{u})+(\mathbf{u} \cdot \nabla \mathbf{u}) \mathbf{u}] .
$$

The density gradient terms from $\partial_{t_{0}} \mathbf{\Pi}^{(0)}$ in (56) are no longer cancelled by density gradient terms from $\nabla \cdot \mathbf{Q}^{(0)}$, and we are missing the uu $\partial_{t_{0}} \rho$ term from $\partial_{t_{0}}(\rho \mathbf{u u})$ that allowed the previous error term to be written as the divergence $\nabla \cdot(\rho \mathbf{u u u})$. The latter missing term is $O\left(\mathrm{Ma}^{4}\right)$, but the $\nabla \rho$ terms are $O\left(\mathrm{Ma}^{3}\right)$, the same order as the previous cubic error term. These pseudoincompressible equilibria therefore pose a much less tractable starting point for obtaining a Galilean-invariant algorithm than the standard compressible equilibria.

\section{Acknowledgments}

It is a pleasure to acknowledge the hospitality of the Isaac Newton Institute programme Partial Differential Equations in Kinetic Theories, and of the Beijing Computational Science Research Center, where parts of this work were carried out. The computations employed the University of Oxford's Advanced Research Computing facilities, the Emerald GPU cluster of the e-Infrastructure South Centre for Innovation, and the fast Fourier transform library FFTW3 [51]. The author's research was supported by an Advanced Research Fellowship from the United Kingdom Engineering and Physical Sciences Research Council [grant number EP/E054625/1]. 


\section{References}

[1] Chapman, S., Cowling, T.G.. The Mathematical Theory of Non-Uniform Gases. Cambridge: Cambridge University Press; 3rd ed.; 1970.

[2] Cercignani, C.. The Boltzmann Equation and its Applications. New York: Springer; 1988.

[3] Koelman, J.M.V.A.. A simple lattice Boltzmann scheme for Navier-Stokes fluid flow. Europhys Lett 1991;15:603-607.

[4] Chen, S., Chen, H., Martínez, D.O., Matthaeus, W.H.. Lattice Boltzmann model for simulation of magnetohydrodynamics. Phys Rev Lett 1991;67:3776-3779.

[5] Qian, Y.H., d'Humières, D., Lallemand, P.. Lattice BGK models for the Navier-Stokes equation. Europhys Lett 1992;17:479-484.

[6] Qian, Y.H., Orszag, S.A.. Lattice BGK models for the Navier-Stokes equation: Nonlinear deviation in compressible regimes. Europhys Lett 1993;21:255-259.

[7] He, X., Luo, L.S.. Theory of the lattice Boltzmann method: From the Boltzmann equation to the lattice Boltzmann equation. Phys Rev E 1997;56:68116817.

[8] Chen, Y., Ohashi, H., Akiyama, M.. Thermal lattice Bhatnagar-Gross-Krook model without nonlinear deviations in macrodynamic equations. Phys Rev E 1994;50:2776-2783.

[9] Weimar, J.R., Boon, J.P.. Nonlinear reactions advected by a flow. Physica A 1996;224:207-215.

[10] Nie, X.B., Shan, X., Chen, H.. Galilean invariance of lattice Boltzmann models. Europhys Lett 2008;81:34005.

[11] Qian, Y.H., Zhou, Y.. Complete Galilean-invariant lattice BGK models for the Navier-Stokes equation. Europhys Lett 1998;42:359-364.

[12] Hazi, G., Kavran, P.. On the cubic velocity deviations in lattice Boltzmann methods. J Phys A 2006;39:3127-3136.

[13] Shan, X., Yuan, X.F., Chen, H.. Kinetic theory representation of hydrodynamics: a way beyond the Navier-Stokes equation. J Fluid Mech 2006;550:413-441.

[14] Chikatamarla, S.S., Karlin, I.V.. Entropy and Galilean invariance of lattice Boltzmann theories. Phys Rev Lett 2006;97:190601.

[15] Yudistiawan, W.P., Kwak, S.K., Patil, D.V., Ansumali, S.. Higher-order Galilean-invariant lattice Boltzmann model for microflows: Single-component gas. Phys Rev E 2010;82:046701.

[16] Dellar, P.J.. Nonhydrodynamic modes and a priori construction of shallow water lattice Boltzmann equations. Phys Rev E 2002;65:036309.

[17] Swift, M.R., Osborn, W.R., Yeomans, J.M.. Lattice Boltzmann simulation of nonideal fluids. Phys Rev Lett 1995;75:830-833.

[18] Holdych, D.J., Rovas, D., Georgiadis, J.G., Buckius, R.O.. An improved hydrodynamics formulation for multiphase flow lattice-Boltzmann models. Int J Mod Phys C 1998;09:1393-1404.

[19] Inamuro, T., Konishi, N., Ogino, F.. A Galilean invariant model of the lattice Boltzmann method for multiphase fluid flows using free-energy approach. Comput Phys Commun 2000;129:32-45.

[20] Wagner, A., Li, Q.. Investigation of Galilean invariance of multi-phase lattice Boltzmann methods. Physica A 2006;362:105-110.

[21] Junk, M., Klar, A.. Discretizations for the incompressible Navier-Stokes equations based on the lattice Boltzmann method. SIAM J Sci Comput 2000;22:1-19.

[22] Wagner, A.J., Yeomans, J.M.. Phase separation under shear in two-dimensional binary fluids. Phys Rev E 1999;59:4366-4373.

[23] Hazi, G., Jimenez, C.. Simulation of two-dimensional decaying turbulence using the "incompressible" extensions of the lattice Boltzmann method. Comput \& Fluids 2006;35:280-303.

[24] Keating, B., Vahala, G., Yepez, J., Soe, M., Vahala, L.. Entropic lattice Boltzmann representations required to recover Navier-Stokes flows. Phys Rev E 2007;75:036712.

[25] Higuera, F.J., Succi, S., Benzi, R.. Lattice gas dynamics with enhanced collisions. Europhys Lett 1989;9:345-349.

[26] d'Humières, D.. Generalized lattice-Boltzmann equations. In: Shizgal, B.D., Weaver, D.P., editors. Rarefied Gas Dynamics: Theory and Simulations; vol. 159 of Prog. Astronaut. Aeronaut. Washington, D.C.: AIAA; 1994, p. 450-458.

[27] Lallemand, P., Luo, L.S.. Theory of the lattice Boltzmann method: Dispersion, dissipation, isotropy, Galilean invariance, and stability. Phys Rev E 2000;61:6546-6562.

[28] d'Humières, D., Ginzburg, I., Krafczyk, M., Lallemand, P., Luo, L.S.. Multiple-relaxation-time lattice Boltzmann models in three dimensions. Phil Trans R Soc Lond A 2002;360:437-451.

[29] Dellar, P.J.. Incompressible limits of lattice Boltzmann equations using multiple relaxation times. J Comput Phys 2003;190:351-370.

[30] d'Humières, D., Bouzidi, M., Lallemand, P.. Thirteen-velocity three-dimensional lattice Boltzmann model. Phys Rev E 2001;63:066702.

[31] Dellar, P.J.. Lattice Boltzmann formulation for Braginskii magnetohydrodynamics. Comput Fluids 2011;46:201-205.

[32] Bhatnagar, P.L., Gross, E.P., Krook, M.. A model for collision processes in gases. I. Small amplitude processes in charged and neutral one-component system. Phys Rev 1954;94:511-525.

[33] Grad, H.. Asymptotic theory of the Boltzmann equation. Phys Fluids 1963;6:147-181.

[34] He, X., Luo, L.S.. Lattice Boltzmann model for the incompressible Navier-Stokes equation. J Statist Phys 1997;88:927-944.

[35] Dellar, P.J.. An interpretation and derivation of the lattice Boltzmann method using Strang splitting. Comput Math Applic 2013;65:129-141.

[36] He, X., Chen, S., Doolen, G.D.. A novel thermal model of the lattice Boltzmann method in incompressible limit. J Comput Phys 1998;146:282-300.

[37] Strang, G.. On the construction and comparison of difference schemes. SIAM J Numer Anal 1968;5:506-517.

[38] Minion, M.L., Brown, D.L.. Performance of under-resolved two-dimensional incompressible flow simulations, II. J Comput Phys 1997;138:734-765.

[39] Chun, B., Ladd, A.J.C.. Interpolated boundary condition for lattice Boltzmann simulations of flows in narrow gaps. Phys Rev E 2007;75:066705.

[40] Kandhai, D., Koponen, A., Hoekstra, A., Kataja, M., Timonen, J., Sloot, P.M.A.. Implementation aspects of 3D lattice-BGK: boundaries, accuracy, and a new fast relaxation method. J Comput Phys 1999;150:482-501.

[41] Mei, R., Shyy, W., Yu, D., Luo, L.S.. Lattice Boltzmann method for 3-D flows with curved boundary. J Comput Phys 2000;161:680-699.

[42] Mayer, G., Házi, G.. Direct numerical and large eddy simulation of longitudinal flow along triangular array of rods using the lattice Boltzmann method. Math Comput Simul 2006;72:173-178.

[43] White, A.T., Chong, C.K.. Rotational invariance in the three-dimensional lattice Boltzmann method is dependent on the choice of lattice. J Comput Phys 2011;230:6367-6378.

[44] Geller, S., Uphoff, S., Krafczyk, M.. Turbulent jet computations based on MRT and Cascaded Lattice Boltzmann models. Comput Math Applic 2013;65:1956-1966.

[45] Kang, S.K., Hassan, Y.A.. The effect of lattice models within the lattice Boltzmann method in the simulation of wall-bounded turbulent flows. J Comput Phys 2013;232:100-117.

[46] Taylor, G.I., Green, A.E.. Mechanism of the production of small eddies from large ones. Proc R Soc Lond A 1937;158:499-521.

[47] Orszag, S.A.. Numerical simulation of the Taylor-Green vortex. Lecture Notes in Computer Science 1974;11:50-64.

[48] Ladd, A.J.C.. Numerical simulations of particulate suspensions via a discretized Boltzmann equation. Part 1. Theoretical foundation. J Fluid Mech 1994;271:285-309.

[49] Dellar, P.J.. Bulk and shear viscosities in lattice Boltzmann equations. Phys Rev E 2001;64:031203.

[50] Chorin, A.J.. A numerical method for solving incompressible viscous flow problems. J Comput Phys 1967;2:12-26.

[51] Frigo, M., Johnson, S.G.. The design and implementation of FFTW3. Proc IEEE 2005;93:216-231. 